\title{
INTERthesis
}

Revista

Internacional

Interdisciplinar INTERthesis

\section{SISTEMAS AGROALIMENTARIOS LOCALIZADOS. INNOVACIÓN Y DEBATES DESDE AMÉRICA LATINA ${ }^{1}$}

\author{
Gerardo Torres Salcido ${ }^{2}$
}

\section{Resumen}

En este artículo se hace una reflexión sobre los problemas que enfrenta la planificación del desarrollo local y sobre las críticas hacia la localización espacial de las actividades socioeconómicas, lo cual es extensivo al estudio de los sistemas agroalimentarios localizados (SIAL). Más adelante, se centra la discusión en la aportación latinoamericana al enfoque SIAL, con base en una perspectiva ética que aborda la visión humanista sobre la seguridad alimentaria desde el contexto de la relación global-local.

Palabras-clave: Sistemas agroalimentarios localizados. Desarrollo territorial. Acción colectiva. Desigualdad social. América Latina.

\section{Introducción}

Los Sistemas Agroalimentarios Localizados (SIAL) es un enfoque de estudio que surge en Francia como producto de la observación de nuevos fenómenos y tendencias en el desarrollo rural, así como del avance del saber e interacción de disciplinas como la Sociología Industrial, la nueva Geografía Humana, la Antropología y la Agronomía. El objeto prioritario de este enfoque es la vinculación profunda que existe entre los territorios y los alimentos. En 1996, un equipo

\footnotetext{
${ }^{1}$ Este artigo foi apresentado oralmente no VI Congresso Internacional Sistemas Agroalimentares Localizados - os SIAL face às oportunidades e aos desafios do novo contexto global em maio de 2013, na cidade de Florianópolis, SC, Brasil.

2 Doctor en Sociologia de la Universidad Nacional Autónoma de México, México. Pesquisador del Centro de Investigaciones Interdisciplinarias en Ciencias y Humanidades (CEIICH) y Coordinación de Humanidades en la Universidad Nacional Autónoma de México, México. Se gradece el apoyo en la edición Elizabeth Jiménez. Este artículo es un producto del proyecto IN 301708 y CONACYT Ciencia Básica 80333. E-mail: tsalcido@unam.mx
}

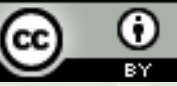
Esta obra foi licenciada com uma Licença Creative Commons - Atribuição 3.0 Não Adaptada. 
coordinado por José Muchnik definió los SIAL como "una concentración de empresas destinadas a la producción, la transformación, el turismo, la gastronomía y los servicios en una escala espacial dada" (MUCHNIK, 1996, p. 5). Esta definición inicial ha cedido su paso a un intenso debate que ha transitado por diferentes momentos, caracterizados todos ellos por el enriquecimiento de las dimensiones de estudio del hecho alimentario. Entre otras, pueden mencionarse la importancia del territorio, la cultura alimentaria, la acción colectiva, la agroindustria rural, el desarrollo de las capacidades, las redes de conocimiento e innovación local, la certificación de la calidad de los productos y las políticas públicas de desarrollo regional y local (MUCHNIK; CAÑADA; SALCIDO, 2008, CAÑADA; VÁZQUEZ, 2005, BOUCHER, 2006, REQUIER-DESJARDINS, 2006).

Las primeras evidencias empíricas de esos sistemas surgieron de la observación de las cadenas de valor "filières" de productos tradicionales como el queso, las viandas y el vino. La concentración de empresas y la cultura alimentaria pronto se relacionaron con las características del territorio. Dadas las evidencias en el ámbito europeo - sobre todo mediterráneo - de esos sistemas y su papel en el desarrollo de las economías locales, el interés creciente que ha despertado su estudio es la pertinencia de elaborar políticas públicas de apoyo, impulso y consolidación de los SIAL a partir de los mecanismos de cooperación internacional, nacional y sub-nacional. Para América Latina y el Caribe, este interés puede ser fundamental para el desarrollo. En este sentido, los propósitos centrales de este artículo son poner en la agenda pública la pertinencia del enfoque SIAL para encontrar caminos alternativos a las políticas sociales en el ámbito rural, basadas casi exclusivamente en transferencias monetarias para combatir la pobreza; así como resaltar algunas temáticas específicas que surgen desde esta región tomando en cuenta las características que le son propias, tales como la pobreza rural, la inequitativa distribución del ingreso y del poder, la seguridad alimentaria y las actividades complementarias que emprenden los hogares rurales para allegarse ingresos no agrícolas, en contextos de diversidad biológica y cultural. Debe señalarse que el contenido de este artículo es de carácter general, de tipo teórico, y aunque recoge algunas de las experiencias de estudio en Europa y América Latina, no expone un estudio de caso o experiencias empíricas particulares.

Hecha esta salvaguarda, la hipótesis que se sostiene en este artículo es que el enfoque SIAL es un método innovador para abordar el desarrollo rural y local en 
las condiciones actuales. Consiste en la formulación de una red de conceptos que sientan las bases para comprender las concentraciones de empresas y las actividades de organización y asociación en torno a productos alimentarios con origen en territorios específicos. Dicha red conceptual expresa la reestructuración de la geografía de la producción y el consumo. Puede considerarse como una reacción a la crisis del consumo de masas y las amenazas a la inocuidad alimentaria, surgidas de las grandes fábricas de alimentos (carnes contaminadas con encefalitis espongiforme, o mal de las vacas locas, vegetales contaminados con Escherichia coli, Salmonella, etc.). Al mismo tiempo, expresa nuevas formas de organización social y la creciente importancia de instituciones locales de innovación tecnológica. No debe confundirse con otros enfoques tales como la "nueva ruralidad" o el de "cadenas de valor". El enfoque SIAL estudia sistemas de producción y consumo con una alta determinación de este último polo. El consumo impone exigencias de calidad, verificaciones sobre el origen de los alimentos y de su identidad territorial, etc. Ambos factores tienen efectos diversos y hasta contradictorios en la organización de la producción.

Como una hipótesis subsidiaria, se sostiene que América Latina aporta al debate del enfoque SIAL una visión propia de acuerdo a la concentración de empresas y agroindustrias destinadas a distribuir una diversidad de productos locales en territorios específicos. Sin embargo, a diferencia de los SIAL europeos, los estudios y observaciones muestran que en nuestra región, esas concentraciones empresariales tienen grandes dificultades para construir economías de escala (tal vez el tequila sea una excepción, pero adolece de una escasa participación de los agaveros en el proceso de definición de las normas de calidad, dominado por las grandes empresas); una deficiente constitución de organizaciones de productores para regular y auto-gestionar la calidad y certificación de los productos; y, en fin, una escasa coordinación de los gobiernos nacionales y locales para llevar a cabo políticas innovadoras. Todo ello en detrimento de la gobernanza de estos sistemas y con graves externalidades sobre el medio ambiente. Esta situación encuentra su base en las realidades del subcontinente dominadas por una agenda en la que destacan con urgencia problemas como la pobreza rural, la seguridad alimentaria, el deterioro de suelos, agua y bosques, los dilemas de la acción colectiva y la ética en los procesos de gobierno, entre otros. 
$\mathrm{Si}$ se toman en cuenta estas particularidades, el enfoque puede ser interesante para su apropiación e innovación en el área de las políticas públicas. En este sentido, el método para escribir este ensayo se basa en una investigación bibliográfica sobre el marco teórico en el que se inserta el concepto de los SIAL, a partir de una pretensión heurística de ubicar la génesis del concepto en el conjunto de los conocimientos sobre el desarrollo territorial. El objetivo es abordar las críticas al desarrollo territorial, al desarrollo local, pero sobre todo, a los sistemas productivos locales y su capacidad de generar alternativas. Este punto de partida es necesario, en la medida que consideramos que los SIAL se encuentran inmersos en el debate sobre la pertinencia de desarrollar los territorios. El método puede resultar útil si logra encontrar puntos de conjunción y comparación, temáticas y asuntos que identifican y bifurcan dicho enfoque en Europa y América Latina para no generar y reproducir visiones de "moda", dogmáticas o acríticas que tanto daño han hecho a las ciencias sociales en el pasado.

Este artículo se divide en tres grandes apartados. En el primero de ellos, se aborda críticamente la literatura de los sistemas locales de producción en el contexto del desarrollo local. A partir de este debate, se especifica, en la segunda parte, el concepto SIAL y su valor epistemológico. En el tercer apartado, se presentan algunas especificidades de esos sistemas en América Latina -con toda la complejidad que esta región tiene- y que consideramos importante resaltar, para dotar a este enfoque de sentido desde nuestra propia realidad. Debe precisarse que este estudio no es definitivo ni generalizable, pues se limita a observaciones puntuales y a la bibliografía sobre el tema. Finalmente, en las conclusiones, se discuten las hipótesis y el método.

\section{Desarrollo territorial y Sistemas Locales de Producción. La crítica y el debate conceptual}

En este apartado se parte del supuesto de que los SIAL son una forma específica de los Sistemas Locales de Producción y que se encuentran ligados al desarrollo de los territorios y los mercados locales. Cabe señalar que en los últimos quince años el desarrollo territorial se ha convertido en un referente para los estudios sobre las áreas rurales (MANZANAL, 2006, MÉNDEZ, 2006, DE JANVRY; SADOULET, 2004, GOVERNA; SALONE, 2004, BOISIER, 1996). Desde nuestro 
punto de vista, son varios los factores que han propiciado la emergencia de este enfoque. Entre otros, pueden ser mencionados los siguientes:

De particular importancia para México y América Latina ha sido la identificación de los territorios con una idea romántica del origen, lo singular, lo específico y la identidad. Un lugar al que se siente arraigo o añoranza y que al separarse de él, en sentido real o metafórico, da pie a la nostalgia. Es decir, a la melancolía que obstaculiza la visión de futuro y de crecimiento humano. Un ejemplo notable, en el caso mexicano, es aportado por Roger Bartra para quien la identidad, ligada a un territorio imaginario y construido por las élites intelectuales, se traduce en una visión nostálgica de los orígenes y una falta de madurez para tomar decisiones y aceptar responsabilidades; es decir, en una incapacidad de madurar (BARTRA, 1987).

Otro factor más, se relaciona con el desarrollo de las teorías de la economía espacial y de la localización, preocupadas por el papel del espacio y la distancia en la economía nacional. Estas teorías tuvieron un gran impulso en las naciones centroeuropeas, sobre todo en Alemania, aunque fueron ignoradas por la mainstream de la teoría económica. Las teorías de la economía espacial y la localización desarrollaron diversas propuestas: desde la formación de estados autárquicos basados en la localización de los productos agrícolas, impulsada por von Thünen, hasta la teoría de la ubicación industrial, de Alfred Weber, pasando por la de los anillos concéntricos, consistente en el papel de la distancia de los núcleos productivos respecto a la ciudad central, en la formación de los precios y las decisiones de localizar la planta industrial, de Lunhardt. Ciertamente, estas propuestas fueron ignoradas por la teoría económica clásica de una manera inexplicable, por lo menos hasta la quinta década del siglo XX (BLAUG, 2001, p. 668).

El tercer factor, relacionado con la seguridad alimentaria y sus aristas éticas, se encuentra íntimamente ligado a la necesidad de atender el desarrollo territorial y a la necesidad de satisfacer nuevas formas de consumo caracterizadas por la búsqueda de productos diferenciados y con arraigo territorial, aunque no necesariamente con la autonomía y soberanía de las comunidades locales. La concentración empresarial de la Gran Industria Alimentaria (GIA) ha exigido la estandarización de los productos de consumo masivo. Este fenómeno ha conducido a la pérdida de valor relativo de la producción agrícola y de la autonomía de los agricultores, en favor de la transformación y distribución alimentarias de gran calado. 
No obstante, en principio la GIA es incapaz de satisfacer las nuevas necesidades del consumo, o el origen y proximidad de los productos, en orden de garantizar la disponibilidad, accesibilidad, inocuidad y autenticidad (cuatro aristas de la seguridad) como formas de aversión al riesgo que plantea el consumo masivo.

Esos antecedentes, sobre todo este último, bosquejan el valor del territorio en los ámbitos de las ideas y las políticas de desarrollo; pero en particular, en el del desarrollo local. En este sentido, las teorías de la localización han sido recuperadas de su tradicional aislamiento en un intento por construir marcos explicativos a los nuevos fenómenos de la producción y los mercados locales (MURDOCH; MARSDEN; BANKS, 2000).

Sin embargo, la idea del desarrollo territorial y su trascendencia para el desarrollo local enfrenta tres importantes cuestionamientos que es preciso enfrentar, dada su relevancia para el surgimiento y consolidación de los Sistemas Productivos Locales: Uno de ellos, es el de la falta de alternativas ante la globalización; el segundo, se relaciona con su impacto en la economía nacional y las políticas públicas, medido en términos del Producto Interno Bruto (PIB); y el tercero, se refiere a la insuficiencia epistemológica del concepto de desarrollo local.

- Con respecto al primer cuestionamiento, la falta de alternativas sistémicas y la escasa validez del desarrollo local frente a la globalización, Hinrichs afirma que las economías locales pueden emprender políticas de diferenciación y labelización (sellos de calidad con anclaje territorial), pero desafortunadamente están destinadas al consumo de las clases medias. En tanto nichos de mercado, los grandes circuitos capitalistas tienden a subsumir esos esfuerzos transformando los labeles locales en commodities, pudiendo coexistir sin problemas con el consumo masivo, más orientado a los consumidores menos informados o con menor poder adquisitivo. Los sistemas agroalimentarios locales no pueden sacudirse las relaciones de poder que terminan ejerciendo los consumidores de clase media en busca de lo "auténtico" o distinguido, sobre los productores y consumidores en desventaja, por su propia situación económica (HINRICHS, 2000). La pretensión alternativa de la producción y consumo localizados a la producción estandarizada sería, en realidad, para la corriente crítica del desarrollo local representada por Hinrichs, una re-funcionalización de los circuitos de comercialización de la GIA y la Gran Distribución (GD) para atender a nichos emergentes de mercado. La subsunción de los circuitos cortos y de proximidad materialmente impediría, a los 
sistemas agroalimentarios locales y a los sistemas productivos locales en general, desempeñar un papel protagónico en la construcción de alternativas globales, pues generalmente terminan agrupándose en un localismo defensivo (WINTER, 2003).

- $\quad$ En cuanto a las políticas públicas de base territorial o local, la crítica contiene dos vertientes: la primera consiste en el valor de lo local para las políticas públicas; y la segunda se refiere a la aportación de las actividades locales al PIB. Algunos críticos como Mohan y Stokke advierten sobre el peligro de preferir las teorías más localizadas, empíricas e inductivas del desarrollo local, que se han difundido en las políticas públicas, a las "holísticas" que antes dominaban los paradigmas de las Ciencias Sociales. Igualmente, consideran peligroso el desplazamiento de términos como "empoderamiento" y "participación" hacia los ámbitos locales en las políticas de desarrollo. Ambos autores advierten que estas nuevas tendencias hacia lo local han producido coincidencias entre la "nueva izquierda" y la "nueva derecha" al subestimar el papel de las relaciones de poder y las desigualdades sociales. También advierten que bajo el nuevo paradigma de lo local se ha descuidado el papel de las empresas nacionales y el poderío de las trasnacionales (MOHAN; STOKKE, 2000, FERNÁNDEZ, 2001).

- $\quad$ El tercer y último cuestionamiento se refiere al concepto de desarrollo económico local. Este concepto supone un diamante que implica la coordinación de instituciones locales, acción colectiva, recursos territoriales, instituciones de conocimiento y medioambiente (ALBURQUERQUE, 2004). Para Mohan y Stokke, así como para Abramovay, la falla fundamental del diamante del desarrollo local es que tiende a subestimar las relaciones de desigualdad y de poder entre lo local y lo global (ABRAMOVAY, 2006, MOHAN; STOKKE, 2000).

Ciertamente estas críticas no son fáciles de contrarrestar. La respuesta requiere de una reflexión serena y cuidadosa para recuperar las raíces teóricas de la discusión, por lo que intentaremos en esta ocasión hacer algunas aportaciones al debate y estimular algunas reflexiones.

En cuanto al primer señalamiento relativo a la falta de respuestas sistémicas del desarrollo local a la crisis capitalista global, se sugiere establecer dos niveles de reflexión: la escala de los cambios y las habilidades sociales.

El primer nivel atañe a los "pequeños cambios" que en un subsistema pueden implicar cambios más profundos y amplios en sistemas complejos. El segundo nivel se relaciona con las habilidades sociales aprendidas o adquiridas en el marco 
territorial. Cambios pequeños y hasta imperceptibles, tienen como consecuencia influir en lo que Wallerstein llama la organización del "sistema-mundo" (WALLERSTEIN, 1989). En este sentido, Braudel elige el Mediterráneo como ejemplo para mostrar la complejidad de la relación del territorio con el sistema mundo. Los pequeños y graduales cambios derivados del comercio, impactan otros sistemas. Los intercambios de productos, ganado, plantas y alimentos que desde América y otras partes del mundo llegaron a Europa influyeron decisivamente en una nueva configuración del territorio. Braudel resalta cómo las nuevas relaciones sociales, que surgen en el Mediterráneo de los siglos XVI-XVIII, nutridas por las venas del comercio mundial, instituyen paradójicamente a los actores e instituciones locales (BRAUDEL, 1976).

En esa misma escala, vale la pena hacer referencia a los pequeños logros, a los que Sen llama funcionamientos de los individuos en sociedad, se relacionan con la construcción de marcos institucionales fuertes, que se derivan de la confianza mutua de los actores en los intercambios y los contratos. La inexistencia de las instituciones o su debilidad, pueden derivar en situaciones de inseguridad de todo tipo; y especialmente, alimentaria (SEN, 1981, 1987).

En cuanto al nivel de las habilidades y capacidades sociales, Abramovay (2006) resalta las aportaciones de Fligstein (2001) y la teoría de los campos desarrollada por Bourdieu para comprender el papel de la movilidad y del cambio institucional. Los campos pueden representar la clausura o emergencia de nuevas formas de conocimiento y de producción que, trasladadas a un ámbito territorial, sintetizan la transformación, evolución o consolidación de las instituciones locales. Las habilidades sociales juegan un papel fundamental para inducir a la cooperación y son tanto más efectivas cuanto se desarrollan en un ámbito de proximidad institucional, es decir, de instituciones - como la de la solidaridad o reciprocidad que promueven el fortalecimiento de esas habilidades sociales.

Con relación a la segunda crítica, la relativa a la aportación de las economías locales al Producto Interno Bruto (PIB), se considera sumamente difícil medir el impacto del desarrollo económico local sobre las cuentas nacionales, por lo que las actividades que tienen su origen en las economías familiares y con un radio territorial limitado, son irrelevantes para la construcción de ese indicador.

Para debatir esta última información es factible recuperar la argumentación de Stiglitz, Sen y Fitoussi sobre el PIB. Los autores consideran que este indicador no 
considera al bienestar social y la desigualdad del ingreso. Estas omisiones urgen a emprender la construcción crítica de nuevos indicadores e índices de medición. Las formas de medición alternativa que proponen los autores parten de los ingresos y del consumo, pero como función del bienestar general de las personas y de sus "capacidades humanas". Los ingresos y gastos monetarios no son vistos como un fin en sí mismo, como se haría desde la medición del PIB, sino como medios para alcanzar el desarrollo centrado en las personas, en los seres humanos (STIGLITZ; SEN; FITOUSSI, 2009). Pero ¿por qué se dice que las actividades económicas no atienden al aspecto humano en las condiciones de la sociedad actual? La respuesta a esta pregunta reside en la excesiva formalización de la economía y en la existencia de un mercado autorregulado que ha perdido su arraigo o incrustación (embeddedness) en la vida social. Para fundamentar más ampliamente nuestra respuesta se recurre a la economía substantiva. Polanyi plantea la necesidad de conocer los procesos de arraigo o "incrustación” (embeddedness) y desincrustación (disembeddedness) de la economía en las relaciones sociales; y, por ende, en las instituciones. En los propios términos de Polanyi, la desincrustación de la vida económica de las relaciones sociales, responde a un periodo sumamente corto de la historia de la humanidad que se materializa en la economía de mercado. Pero aún en esta etapa histórica relativamente corta, su predominio es parcial. Las regulaciones e intercambios no descansan exclusivamente en la noción de utilidad económica. Por el contrario, responden a valores tales como la reciprocidad, el reconocimiento y la solidaridad que son comunes en la llamada economía primitiva (POLANYI, 2006, BLOCK, 2003). Valores que, desde el punto de vista de la Sociología Económica, sólo pueden darse en términos de proximidad geográfica y/o institucional.

Relativo a la tercera crítica, que hace énfasis en la abstracción del desarrollo local respecto a las situaciones de desigualdad y las asimetrías de poder, consideramos que el esquema de la "incrustación" puede ser pertinente para el caso del desarrollo económico local. Los territorios pueden poseer, además de activos tangibles, intangibles; es decir, valores e instituciones que favorecen la integración de la economía y la sociedad. Ciertamente, se requiere valorar las capacidades sociales que se expresan en la habilidad de limitar la acción de los agentes nocivos para la acción colectiva, tales como el "free rider" e inducir a la cooperación, pero sin ignorar las diferencias y conflictos en la localidad. Esto último plantea algunos 
problemas de gobernanza tales como la coordinación de las élites rurales, su capacidad de gestión y de conducción de proyectos; su disposición a diagnosticar las oportunidades de comercialización o transformación y la vinculación de la comunidad con las esferas de gobierno o con las empresas; así como la disposición de todos estas instancias a la mayor horizontalidad posible en la toma de decisiones. Por otra parte, plantea problemas éticos, tales como la justicia, la equidad de género y la honestidad, entre otros, que son sumamente importantes en sociedades en situaciones de pobreza y de profunda desigualdad.

En suma, las críticas del desarrollo local y al potencial de los sistemas agroalimentarios o productivos locales son sumamente importantes para plantear alternativas; pero carecen, desde nuestro punto de vista, de una reflexión sobre el papel de la acción colectiva, las instituciones y los valores. Éstos son la arcilla que une a las regulaciones y normas que ponen en funcionamiento intangibles basados en habilidades cooperativas y en mecanismos de resolución de conflictos en un contexto territorial.

\section{La especificidad del SIAL como concepto}

Las reflexiones anteriores son relevantes para abordar la emergencia de los SIAL en América Latina después de la crisis del ajuste neoliberal de los años 80 y 90, como en el caso de México; o de la supeditación profunda de los sistemas agroalimentarios de países como Argentina y Brasil a la producción de commodities, que se ejemplifica en el caso de la soya. Los SIAL pueden considerarse como una forma del desarrollo local, que, no obstante, no corresponden a economías autárquicas, sino a la necesidad de nuevas formas de gobernanza territorial que redundan en la apropiación de valor por parte de los productores y consumidores locales; valor que de otra manera, sería trasladado a otras instancias de la cadena.

La hipótesis de que los SIAL son subsistemas insertos en el Sistema Agroalimentario Global y que representan una estrategia emergente de desarrollo basada en la estructuración de redes, campos y habilidades como partes integrantes de la "inteligencia territorial", es una aportación a la comprensión del enfoque que intenta construir el concepto SIAL (MUCHNIK; CAÑADA; SALCIDO, 2008). Dicha contribución puede ser útil para ubicar las características de la acción social, tales como el manejo de los recursos colectivos, las indicaciones geográficas y la 
valoración de la tipicidad de los bienes de consumo alimenticios, entre otras instituciones a considerar en este apartado, y como alternativas a las críticas al desarrollo territorial.

El reconocimiento de la particularidad de los SIAL y su diferenciación con otros enfoques, tiene varios antecedentes teóricos, aunque se ha reconocido en la literatura la preeminencia del distrito industrial Marshalliano y la visión económica de Karl Polanyi. No obstante, no deben ignorarse otros antecedentes que recogen el debate sistémico sobre la función de la agricultura en las tradiciones anglo-sajona de los Estados Unidos de América (EUA) y europeas mediterráneas. Desde nuestro punto de vista, el enfoque SIAL recoge las influencias de la teoría de los sistemas; de la complejidad y el desarrollo económico local, por medio de la Sociología y la Economía Industriales; pero también de las nuevas tendencias de la Geografía Humana. Ahora bien, dado que el enfoque SIAL no es sólo una posición teórica sino de intervención social, también potencia las teorías de la acción social y colectiva en el lugar, el territorio. Esta segunda cuestión ha llevado a una discusión sobre las formas de activación de los sistemas agroalimentarios locales, aunque también ha abierto la puerta a un tercer momento del debate sobre el concepto SIAL en el que se entiende a éste como un subsistema inserto en un sistema territorial más amplio, en el que es posible encontrar y estudiar no sólo un producto, sino una multitud de los mismos. En este sentido hemos pasado de la caracterización de los SIAL, a la activación de los mismos y a su inserción en territorios complejos en los que un SIAL es un nodo más, muy importante, por cierto, del desarrollo. En este apartado se recorren estos tres momentos de la construcción del concepto y se reflexiona sobre sus perspectivas.

El enfoque sistémico se desarrolla a partir de los años cincuenta del siglo pasado. Es notable que hasta mediados de esa década, la economía agroalimentaria se limitaba a estudiar la agricultura desde el ángulo de la oferta. Sin embargo, Davis y Goldberg (1957) Ilamaron la atención sobre la creciente integración entre agricultura, industria y comercialización que se desarrolla en torno a la explotación agrícola y sus efectos sistémicos en otras actividades (SALCIDO, 2009). Este proceso fue llamado agribusiness. Las aportaciones de Davis y Goldberg propiciaron el crecimiento de los estudios sobre la agricultura y las áreas rurales desde otras perspectivas de interés económico. Esta visión, en gran medida, 
coincide con los estudios que Bertalanffy venía desarrollando sobre la teoría de los sistemas de una manera más ambiciosa desde inicios de esa década.

Con todo y que el análisis de la producción agrícola en sus interacciones con la industria y la comercialización se dieron en el contexto de aumentar la productividad y la integración en los mercados globales, el concepto de agribusiness planteó en términos metodológicos la utilidad de una noción que en la literatura francesa adquiriría el nombre de "filière" (MALASSIS; PADILLA, 1979) entendido como la coordinación de un encadenamiento productivo caracterizado por una concentración empresarial en el territorio cuyo objetivo es el aumento y la apropiación de valor.

Las raíces de los Sistemas Agroalimentarios Locales como sistemas complejos se derivan de las circunstancias mismas de incertidumbre en la que se mueve el Sistema Agroalimentario Global, de sus interacciones con los sistemas locales y de su constante retroalimentación. Asimismo, se refieren a la constante individualización de los agentes territoriales que inducen a nuevos riesgos y formas de adaptación, que tienen como consecuencia la reestructuración de los sistemas. La complejidad no sólo se plantea en términos de los sistemas económicos y a su incrustación en la vida social, sino que más ampliamente se refiere a los vínculos eco-sociales y las consecuencias de las externalidades medioambientales, por lo cual es pertinente la conformación de una serie de valores nuevos en torno a la relación entre desarrollo, conservación y medio ambiente, retomando la idea de una ética compleja que se abordará más adelante (MORIN, 2008).

Las raíces de la Sociología Económica y la Geografía Humana son más amplias en la literatura de los SIAL, aunque no están exentas de la complejidad. En un plano histórico, la reestructuración de los sistemas agroalimentarios en las décadas de los ochenta y noventa del siglo XX se inicia con la crisis de lo que se ha dominado "fordismo alimentario" caracterizado por una saturación y abundancia de los productos alimenticios estandarizados (BONANO, 2007, BAGNASCO, 1997, CAÑADA, 1997). Sin embargo, paradójicamente, la saturación del consumo marca al mismo tiempo la posibilidad de la valoración de los productos específicos. Esta valoración ha estado determinada, en gran medida, por la incertidumbre provocada por amenazas a la seguridad e inocuidad alimentarias; al medio ambiente como resultado de la creciente contribución de los sistemas agropecuarios a la emisión de gases de efecto invernadero; a la coordinación del sistema (crisis de las relaciones 
verticales, cambio en las motivaciones de los empleados, reclamos de productores y consumidores) y la emergencia de un modelo basado en la demanda, es decir, en las exigencias del consumidor.

Retomando los antecedentes teóricos, curiosamente la literatura de los SIAL no hace referencia a la teoría económica de la localización, pero sí reconoce a un teórico de la utilidad marginal por sus estudios sobre el papel de la industria en la distribución de costos y beneficios. En Industry and Trade, Marshall (1920) constata la existencia de economías de aglomeración en las zonas industriales de Inglaterra. Las ventajas de estas economías no se limitan a la disminución de los costes de transportación, sino que expresan un incremento en las capacidades de innovación debido a la cooperación entre las empresas y otros agentes del territorio; es decir, de intangibles que pueden erigirse en la base para incrementar los beneficios de las empresas en esos territorios.

Las hipótesis de Marshall respecto al papel de los intangibles en las concentraciones empresariales fueron ignoradas por lo menos hasta los años ochenta y noventa del siglo XX, cuando representantes de la sociología económica italiana como Becattini, Brusco y Bagnasco rescataron la idea de los distritos industriales de Marshall por su potencialidad de explotación de las capacidades sociales y la cooperación institucional (BECATTINI, 2004, ARCANGELI; BIANCHI; MAGNANI, 1985, BAGNASCO et al., 1993, PYKE et al., 1992). Para la escuela de la Sociología Industrial italiana, la competitividad de Italia y su desarrollo económico se explican por una fuerte identidad territorial y los lazos de cooperación y confianza para la difusión de las innovaciones en un contexto de pequeñas y medianas empresas enlazadas en redes sociales y familiares.

No obstante el éxito de los hallazgos de los distritos industriales italianos, se puso de manifiesto que el modelo podría tener serias dificultades para replicarse en otras partes del mundo. Rabellotti puso en duda la existencia de un modelo de "distrito industrial" para el caso de la industria del calzado con base en una comparación de los distritos de Brenta y Marche en Italia, y de León y Guadalajara, en México; aunque encuentran ventajas en la cooperación y difusión de adelantos tecnológicos para las pequeñas empresas, sobre todo en el caso de México (RABELLOTTI, 1995).

Schmitz encontró, a propósito de la industria del calzado, que si bien podrían existir características comunes en los distritos o "arranjos" productivos en el caso de 
Brasil, que identificaban las actividades de las pequeñas empresas con el caso italiano, la importancia de las relaciones sociales y los intercambios entre las empresas se subordinaban a los papeles especializados asignados por la gran empresa de carácter mundial. De este modo, más que un distrito industrial, lo que convenía era caracterizar estas actividades como cluster, economías localizadas y altamente especializadas bajo las directrices de la gran empresa, aunque su base consistiera en un saber hacer en los territorios a lo largo de generaciones (SCHMITZ, 1995). Para Porter, los cluster representan una nueva forma de economía que está dirigida a potenciar las ventajas competitivas de las regiones (PORTER, 1998).

Otra de las vertientes que señalan el origen de los SIAL, es la nueva Geografía Humana y la Teoría del Desarrollo Rural. Paul Krugman ha defendido la idea de que el territorio es el "lugar" marcado y determinado por las relaciones sociales, la impronta de la actividad humana y la identidad de los habitantes y con ello ha dado un nuevo marco al estudio de los territorios y sus interacciones económicas y sociales. En este sentido, la obra de Krugman ha dado una particular atención a las dinámicas culturales e institucionales en los territorios y su papel en la definición de la diferenciación y especialización de las regiones (FUJITA et al., 1999, KRUGMAN, 1995).

Una de las razones de ese creciente interés, se debe a la existencia de lo que se llamó Sistemas Productivos Localizados (SPL) - los que no se limitan a los alimentos, sino que incluyen las artesanías, otros productos como el calzado, el vestido o inclusive alta tecnología, como la producción de software- en los que interactúan las capacidades sociales con otros mecanismos de funcionamiento económico como la difusión de nuevos inventos, artefactos o técnicas relevantes en la industria o la administración que implican procesos colectivos de apropiación y difusión del saber.

La idea de activar los SIAL surge de las capacidades individuales y colectivas en territorios específicos. Se parte del supuesto de que en los territorios rurales existen recursos naturales o culturales cuya combinación ha llevado al desarrollo de formas de apropiación y transformación de los productos que deben ser puestos en valor para lograr el bienestar de las comunidades rurales. En el caso de América Latina, la activación de los SIAL tiene relevancia para la construcción de alternativas a la pobreza rural en función de una mayor apropiación del valor de la producción, 
ya sea mediante la transformación industrial a pequeña escala, o bien mediante actividades que ofrezcan servicios ambientales y turísticos.

Una primera cuestión que se encuentra inmersa en la activación de las capacidades es que los SIAL responden a la declinación de los ingresos agrícolas. Las estrategias de incrementar los ingresos por parte de los hogares rurales responden a la multifuncionalidad creciente del campo, ya que no sólo se le considera, por los nuevos consumidores y las políticas públicas, como productor de alimentos, sino como depositario de tradiciones y cultura culinaria, así como proveedor de servicios ambientales y turísticos a las áreas urbanas valorizando el patrimonio paisajístico y cultural. Las particularidades enunciadas imprimen sellos a la Agroindustria Rural (AIR) como alternativa. François Boucher y Denis Requier Desjardins han sido pioneros en este aspecto al estudiar las queserías rurales de Cajamarca en Perú, y otros sistemas agroalimentarios locales en Colombia o Costa Rica. La pregunta fundamental que ha guiado este momento en el debate del SIAL ha sido justamente cómo activar las capacidades de los territorios y cómo pasar de la observación y caracterización de estos sistemas a las políticas y programas públicos de desarrollo.

Uno de los problemas que han sido abordados en este plano es el relativo a la acción colectiva, los ambientes institucionales y el liderazgo. De particular importancia en los procesos de activación ha sido cómo controlar y disminuir las consecuencias de la existencia de los "free riders" o de los liderazgos que tienden a obstaculizar, y no a facilitar, la gestión. Unido a estos dos problemas se encuentra el deficiente acceso a los bienes públicos gubernamentales. En este sentido, Courlet (2002) afirma que el concepto mismo de SIAL ha tenido una evolución, lo que denota su apertura epistemológica, cuya flexibilidad le permite incorporar otros conceptos y enfoques como el capital social y la gobernanza.

Hasta aquí, la literatura ha definido algunas características comunes de los SIAL. Brevemente, dichas características son:

- $\quad$ la existencia de uno o varios productos locales;

- la existencia y consolidación de instituciones que faciliten el desarrollo de la Agroindustria Rural (AIR) con base en la confianza y los acuerdos;

- $\quad$ prácticas sociales de innovación y difusión;

- $\quad$ capacitación y apoyo tecnológico generados por los productores o por asociaciones de carácter gubernamental o no gubernamental;

R. Inter. Interdisc. INTERthesis, Florianópolis, v.10, n.2, p. 68-94, Jul./Dez. 2013 
- desarrollo y defensa de la calidad, tomando en cuenta el consumo urbano y las exigencias sanitarias;

- $\quad$ generación y apropiación de valor;

- disminución de los costes de transacción, la incorporación de patrones de calidad y de la protección de las denominaciones geográficas y de origen; y

- $\quad$ disminución de las externalidades medioambientales negativas.

En un tercer momento de la evolución del concepto de la discusión conceptual de los SIAL, se manifiesta un incipiente retorno a las bases territoriales y locales del desarrollo. La multifuncionalidad de las áreas rurales y las características de los sistemas agroalimentarios locales, enunciadas más arriba, sientan las bases para una conceptualización de los SIAL y su interacción con sistemas más amplios de producción y consumo con base en la definición de los territorios, la acción colectiva y las instituciones. Estudios recientes en América Latina muestran una fuerte interrelación entre la especialización y localización de esos sistemas y otras actividades que derivan de la multifuncionalidad propia de las áreas rurales, multifuncionalidad que adquiere una importancia mayor. En las comunidades centrales de México, las dificultades de acceso a los bienes públicos, conflictos causados por la asimetría en la distribución del poder o por la desigual distribución y acceso a recursos comunes, como por ejemplo el agua, los centros de acopio comunitario o el crédito, han llevado a las comunidades a explotar más ampliamente la diversidad biótica y los recursos territoriales aunque enfrenten problemas institucionales, que no son tan fuertes y comunes en Europa. Ejemplos en este sentido han sido mostrados en otros libros ya citados en este artículo o informes como el de Boucher, Requier y Brun (2010) sobre la agroindustria rural en la Selva Lacandona, en Chiapas.

La revisión de estos tres momentos del concepto debe considerarse como una reflexión crítica que deja abiertas muchas posibilidades de continuar el debate. No debe considerarse como un tema clausurado o una visión evolucionista en la cual el concepto SIAL aparece como culminación de una serie de desarrollos previos. El debate, como todo argumento, puede tener múltiples salidas y aperturas. Precisamente en este sentido es que se ha insistido en la capacidad de innovación que presenta este concepto y en su trascendencia. Por ello, es preciso reflexionar sobre la hipótesis subsidiaria establecida al inicio de este artículo. 


\section{Pobreza, desigualdad y valores de la acción colectiva. Retos a superar en América Latina}

Como se ha visto en los párrafos anteriores, la conceptualización de los sistemas agroalimentarios locales adquiere valor epistemológico por ser una herramienta que pretende explicar los fenómenos emergentes de resistencia de los actores locales - productores y consumidores- al predominio de la producción fordista y al consumo de masas. Por otra parte, este proceso corresponde a una necesidad de diferenciación del consumo, de exigencia por la calidad y de identidad territorial de los alimentos. Existen, sin embargo, otros elementos que han surgido en la agenda propiamente de América Latina. Algunos de estos elementos son los siguientes: en primer lugar, la pobreza rural; en segundo lugar, las particularidades de la acción colectiva en América Latina y en tercer lugar, el sistema institucional y los valores.

Los SIAL como alternativas a la pobreza rural se diferencian de la conceptualización original de los colegas europeos porque en Europa el problema de los sistemas agroalimentarios locales consiste en garantizar la calidad, las normas de origen y la protección del territorio en función de la integración vertical y horizontal de los procesos de producción y consumo, lo que implica reunir una serie de características de organización social e institucionalidad. Los SIAL en América Latina difícilmente conjuntan esas características. Requieren de elecciones e inversión; reconocimiento y valorización del saber hacer; una institucionalidad que facilite los procesos de transformación y comercialización de los productos, y un marco de difusión de las innovaciones (BOUCHER; CARIMENTRAND; REQUIERDESJARDINS, 2003). No obstante, existen algunos ejemplos razonablemente exitosos, como los casos de producción de nopal en Tlalnepantla, Morelos, México; de hortalizas y hongos cuitlacohe de invernadero en Tlaxcala y otros, que han conjuntado una serie de elementos como los siguientes:

- $\quad$ explotación de la identidad territorial del producto;

- introducción de procesos previos o posteriores a la cosecha que marcan el inicio de una agroindustria rural (diseño y construcción de invernaderos en el caso de los productores de Tlaxcala o de empacadoras, deshidratadoras de nopal, en el caso de Tlalnepantla); 
- liderazgos capaces para gestionar y conducir las negociaciones con organizaciones más allá de las comunidades;

- $\quad$ organización social de origen étnico o fuertemente arraigado en las tradiciones indígenas de los campesinos del Centro de México;

- $\quad$ cooperación científica y de instituciones académicas;

- una creciente conciencia ambiental; y

- coordinación y negociaciones con las instancias de gobierno nacional, estatal, pero sobre todo, municipal.

La conjunción de estos factores no implica, sin embargo, que se superen los problemas de pobreza, desigualdad y de asimetría de poder en los ámbitos rurales que se expresan de múltiples maneras: desde la construcción de capital social cerrado, la escasa difusión de las innovaciones, gestiones opacas o carencias en la socialización de información; hasta la exclusión de grupos enteros de los circuitos de comercialización, sin omitir las desigualdades de género. Además de las presiones de mercado, que conducen en general a la especialización y por ende, a la disminución de la diversidad biótica de los territorios, las desigualdades y asimetrías apuntadas son un obstáculo para la seguridad alimentaria entendida como acceso, inocuidad, calidad y conservación del patrimonio gastronómico.

Esto último conduce al planteamiento de la ética y sus funciones en la estructuración de los SIAL. En otra parte, la ética ha sido definida como la capacidad colectiva de habituarse, de construir hábitos y costumbres que suelen diferenciarse de la moral, entendida como norma de comportamiento individual (SALCIDO; CHÁVEZ; LEGLISE, 2011). En este sentido, la ética dirigida al bienestar social es ininteligible si no se ubica en la perspectiva de una situación deseada, que emana de una serie de valores. A menudo se presume que éstos permiten intercambios en el marco de normas universalmente aceptadas y son la base de la generación de identidades que facilitan a las personas, las organizaciones y las instituciones funcionar en torno a metas comunes.

En las sociedades rurales, no obstante, los valores suelen tener un trasfondo de cooperación y conflicto colectivos que determinan las dinámicas locales. Ahora bien, en términos de un concepto SIAL desde América Latina, es importante observar esas dinámicas no sólo por sus impactos en la distribución de los beneficios, generalmente escasos, sino por la apropiación del territorio de parte de 
los diversos agentes. También es importante observarlas por el papel que tienen en la generación de demandas de una mayor participación de la sociedad local en el valor de los productos generados. Asimismo, por el papel que los SIAL pueden tener en las actitudes de conservación y desarrollo de la diversidad biótica, étnica y productiva.

Este último punto nos lleva a valorar las aportaciones de una ética compleja, producto de un pensamiento eco-social como sostiene Morin, a quien ya hemos citado. La minimización del riesgo de daño físico por la carencia prolongada de los alimentos en una hipotética situación en la que el acceso general a los alimentos hubiese sido resuelto como producto de políticas sociales universales, no debe ocultar el hecho de que esa abundancia también puede contener peligros derivados de la contaminación del agua y del aire por el uso de pesticidas y fertilizantes químicos, por lo que se requiere una tecnología adecuada a las características del territorio. Así, en el ámbito de lo que hemos definido como ética se requiere un marco de derechos y obligaciones que permitan disminuir la inseguridad, es decir, la amenaza a la vida o a la integridad física de las personas, pero también de los entornos naturales.

En este sentido, debe enfatizarse que el combate a la desigualdad y la superación de la pobreza, no depende sólo de medidas económicas. De ahí que desde la perspectiva latinoamericana, es vital el fortalecimiento institucional, y la acción social en un contexto de disminución de externalidades negativas. La ética puede corresponder a esa acción colectiva que se materializa en reglas del juego, marcos normativos y cognitivos explícitos e implícitos.

La inclusión de los valores en el proceso de construcción y cambio institucional en un territorio, es fundamental en un esquema de política pública de prevención de riesgos, como por ejemplo, las consecuencias de la inseguridad alimentaria. La intensidad con la que una sociedad construye y asume los valores genera un esquema de cumplimiento de los derechos, pero también de las obligaciones entre las instituciones públicas-gubernamentales, los privados, las organizaciones sociales y de todas estas instancias hacia el entorno ambiental. Se reconoce generalmente que también estimulan la integración, el reconocimiento y el fortalecimiento de la identidad.

\section{Conclusión}

R. Inter. Interdisc. INTERthesis, Florianópolis, v.10, n.2, p. 68-94, Jul./Dez. 2013 
En este artículo se ha cocinado, con ingredientes de diferente procedencia, una reflexión sobre los problemas que enfrentan los SIAL como concepto y experiencia de desarrollo, insertos en los territorios y el desarrollo local. Las hipótesis de las que se ha partido han sido discutidas a lo largo de este artículo. La innovación del enfoque SIAL consiste en su capacidad de integrar la observación empírica de los sistemas agroalimentarios locales y el avance del saber en una serie de disciplinas cuyos objetos de investigación y propuestas confluyen, cada vez más, en el desarrollo territorial. Por ello es posible construir una primera respuesta a la hipótesis alternativa, es decir, a la inevitabilidad de la subordinación de los territorios al espacio global, que enarbolan los críticos de la localización de las actividades económicas. Respuesta que ha sido reforzada por la importancia y el interés que han adquirido los SIAL desde los medios académicos, los productores, las instituciones públicas-sociales y privadas. Las experiencias que han sido estudiadas en contextos europeos y de América Latina, sugieren una marcada tendencia a valorar las experiencias locales y los actos económicos e institucionales de proximidad, por su gran potencial para reconstruir las relaciones sociales y formas de consumo en territorios específicos.

Para darle un sentido a estas formas emergentes y resistentes a la producción y consumo fordistas, se ha llevado a cabo un debate sobre las particularidades de los SIAL en América Latina y las posibilidades de construir una agenda común en esta región. No sólo se trata de ver esos sistemas como un mecanismo de apropiación de valor de las comunidades y de diversificación de ingresos no agrícolas, tampoco se trata de centrarlos exclusivamente en los problemas de la calidad y la certificación; y, ni siquiera, de ver los problemas de la gobernanza para incrementar la utilidad de los productores; se requiere integrar, además de todas esas dimensiones que dominan las preocupaciones originales del concepto SIAL, su significado para las políticas de desarrollo como alternativas a la pobreza rural y a las políticas sociales dominantes. Darles sentido en función de las capacidades de las comunidades para valorar su propio saber hacer. Valorar su autonomía como una base esencial de la seguridad alimentaria. Junto a estas preocupaciones, se ha insistido en integrar la dimensión de los valores para incrementar los mecanismos de confianza, solidaridad y manejo de conflictos en márgenes de tolerancia y democracia. 
De ninguna manera puede afirmarse que la conclusión consiste en las hipótesis demostradas. Su enunciación, y el método relacional de los conceptos que se ha desarrollado en este artículo, apenas nos aproximan a una discusión que debe profundizarse para dotar de sentido a las redes latinoamericanas de investigación con el enfoque SIAL, pero también a las políticas públicas que se deriven de este enfoque en nuestros contextos. 


\section{SISTEMAS AGROALIMENTARES LOCALIZADOS. INOVAÇÃO E DEBATES NA AMÉRICA LATINA}

\section{Resumo:}

Este artigo propõe uma reflexão sobre os problemas enfrentados pelo planejamento do desenvolvimento local e sobre as críticas centradas na localização espacial das atividades econômicas, explorando as implicações desse argumento para o estudo de sistemas agroalimentares localizados (SIAL). Além disso, o autor avalia de que maneira as pesquisas conduzidas em diversos países latino-americanos vêm contribuindo para a maturação progressiva do enfoque SIAL. Para tanto, adota uma perspectiva ética que resgata a visão humanista sobre a problemática da segurança alimentar no atual contexto de globalização econômico-financeira e cultural.

Palavras-chave: Sistemas agroalimentares localizados. Desenvolvimento territorial. Ação coletiva. Desigualdade social. América Latina.

\section{LOCAL AGRI-FOOD SYSTEMS. INNOVATIONS AND DEBATES IN LATIN AMERICA}

\section{Abstract:}

This paper synthesizes a reflection about the challenges related to the planning of local development strategies and specifically to the spatial distribution of economic activities exploring the implications of this argument for the study of local agri-food systems. Moreover, the author evaluates the Latin-American contribution to foster the SIAL approach, from an ethical point of view that focuses the humanist conception of food security in relation to the asymmetries of the globalization process.

Keywords: Local agri-food systems. Territorial development. Collective action. Social inequality. Latin America. 


\section{REFERENCIAS}

ABRAMOVAY, R. Para una teoría de los estudios territoriales. In: MANZANAL, M.; NEIMAN, G.; LATTUADA, M. Desarrollo rural: organizaciones, instituciones y territorios. Buenos Aires: CICCUS, 2006. p. 51-70.

ALBUQUERQUE, F. Desarrollo económico local y descentralización en América Latina. Revista de la CEPAL, n. 82, p. 157-171. 2004.

ARCANGELI, F.; BIANCHI, G.; MAGNANI, I. Sviluppo multiregionale: teorie, metodi, problemi. Milano: Franco Angeli, 1985.

BAGNASCO, A. La función de las ciudades en el desarrollo rural: la experiencia Italiana. Enlace. Abasto y Alimentación, v. 2. n. 8, p. 9-13. 1997.

BAGNASCO, A.; TRIGILIA, C.; BERTHET, T. et al. La construction sociale du marché: le défi de la troisieme Italie.Cachan: Editions de L'Ens-Cachan, 1993.

BARTRA, R. 1987. La jaula de la melancolía. Identidad y metamoforsis del mexicano. México: Grijalbo, 1987.

BECATTINI, G. Industrial Districts: a new approach to industrial change. United Kingdom: Edward Elgar, 2004.

BLAUG, M. Teoría económica en retrosprección. $4^{a}$ ed. México: Fondo de Cultura Económica, 2001.

BLOCK, F. Karl Polanyi the Writing of "The Great Transformation". Theory and Society, v. 32, n. 3, p. 275-306. 2003.

BOISIER, S. Modernidad $y$ territorio. $1^{\text {th }}$ ed. Santiago de Chile: Instituto Latinoamericano y del Caribe de Planificación Económica y Social-ILPES, 1996.

BONANO, A. A Gobalizaçao da economia e da sociedade: fordismo e Pósfordismo no sector agroalimentar. 2007. Disponible en: <http://www2.cddc.vt.edu/digitalfordism/fordism materials/index.htm>. Acceso en: 06 jul. 2007. 
BOUCHER, F. Agroindustria rural y sistemas agroalimentarios locales. Nuevos enfoques de desarrollo territorial. III Congreso Internacional de la Red SIAL. Alimentación y Territorios, ALTER 06, CSIC-Gis-SYAL-Agropolis, Baeza, España. 2006.

BOUCHER, F.; REQUIER-DESJARDINS, D.; BRUN, V. SYAL: Un nouvel outil pour le developpement de territoires marginaux. les leçons de l'alliance des agroindustries rurales de la selva lacandona, Chiapas. Montpellier: Innovation, C.U.M.R, 2010. Disponible en: <http://www.isda2010.net>. Acceso en: 16 jul. 2010.

BOUCHER, F.; CARIMENTRAND, A.; REQUIER-DESJARDINS, D. Agroindustrie rurale et lutte contre la pauvreté: Les Systèmes Agroalimentaires Localisés contribuent-ils au renforcement des "capabilités"? In: DUTEURTRE, G.; FAYE, B. Elevage et pauvreté: actes de l'atelier-recherche CIRAD. Montpellier: CIRAD, 2003.

BRAUDEL, F. El Mediterráneo y el mundo mediterráneo en la época de Felipe II. $2^{a}$ ed. México: Fondo de Cultura Económica, 1976.

COURLET, C. Les systèmes productifs localisés. Un bilan de la literature. Etud. Rech. Syst. Agraires Dev., v. 33, p. 27-40. 2002.

DAVIS, J. H.; GOLDBERG, R. A. A concept of agribusiness. Boston: Division of Research, Graduate School of Business Administration, Harvard University, 1957.

DE JANVRY, A.; SADOULET, E. Hacia un enfoque territorial del desarrollo rural. Preparado para el IV Foro temático Regional de América Latina y el Caribe: Cosechando oportunidades: Desarrollo Rural en el Siglo XXI. Universidad de California (Sede de Berkeley). 2004.

FERNÁNDEZ, V. R. Estrategia(s) de desarrollo regional bajo el nuevo escenario global-local: revisión crítica sobre su(s) potencialidad(es) y límites. EURE (Santiago), v. 27, n. 82, p. 43-63. 2001.

FLIGSTEIN, N. Social Skill and the Theory of Fields. Sociological Theory, v. 19, n. 2, p. 105-125. 2001.

FUJITA, M.; KRUGMAN, P. R.; VENABLES, A. The spatial economy: cities, regions and international trade. Cambridge, Mass.: MIT Press, 1999. 
GOVERNA, F.; SALONE, C. Territories in action, territories for action: The territorial dimension of Italian local development policies. International Journal of Urban and Regional Research, v. 28, n. 4, p. 796-818. 2004.

HINRICHS, C. C. Embeddedness and local food systems: notes on two types of direct agricultural market. Journal of Rural Studies, v. 16, n. 3, p. 295-303. 2000.

KRUGMAN, P. R. Development, Geography, and Economic Theory. Cambridge, Mass.: MIT Press, 1995.

MALASSIS, L.; PADILLA, M. Economie agro-alimentaire. Paris: Editions Cujas, 1979.

MANZANAL, M. Regiones, territorios e institucionalidad del desarrollo rural. In: MANZANAL, M.; NEIMAN, G.; LATTUADA, M. Desarrollo rural. Organizaciones, instituciones y territorio. Buenos Aires: Ciccus, 2006. p. 21-50.

MARSHALL, A. Industry and Trade: A Study of Industrial Technique and Business Organization, and of Their Influences on the Conditions of Various Classes and Nations. London: Macmillan and Co., 1920.

MÉNDEZ, R. Difusión de innovaciones en sistemas productivos locales y desarrollo territorial. III Seminario Internacional de la RED SIAL. Alimentación y Territorios. ALTER, 6 ed., CSIC-Gis-SYAL-Agropolis-Universidad Internacional de Andalucía, Baeza, España. 2006.

MOHAN, G.; STOKKE, K. Participatory development and empowerment: The dangers of localism. Third World Quarterly, v. 21, n. 2, p. 247-268. 2000.

MORIN, E. La méthode. France: Seuil, 2008.

MUCHNIK, J. Systèmes agroalimentaires localisès: organisations - innovations et developpement local. Montpellier: CIRAD - SAR, 1996.

MUCHNIK, J.; CAÑADA, J. S.; SALCIDO, G. T. Systèmes agroalimentaires Localisés: etat des recherches et perspectives. Cahiers Agricultures, v. 17, n. 6, p. 513-519. 2008. 
MURDOCH, J.; MARSDEN, T.; BANKS, J. Quality, Nature, and Embeddedness: Some Theoretical Considerations in the Context of the Food Sector. Economic Geography, v. 76, n. 2, p. 107-125. 2000.

POLANYI, K. La gran transformación. Los orígenes políticos y económicos de nuestro tiempo. $2^{\mathrm{a}}$ ed. México: Fondo de Cultura Económica, 2006.

PORTER, M., 1998. Clusters and the New Economic of Competition. Harvard Business Review, v.76, n. 6, p.77-90, nov./dic. 1998.

PYKE, F.; BECATTINI, G.; SENGENBERGER, W. et al. Los distritos industriales y las pequeñas empresas. Madrid: Centro de Publicaciones, Ministerio de Trabajo y Seguridad Social, 1992.

RABELLOTTI, R. Is there an 'industrial district model'? Footwear districts. World Development, v. 23, n.1, p. 29. 1995.

REQUIER-DESJARDINS, D. Globalización y evolución de la agro-industria rural en los países andinos. Un enfoque sobre los "sistemas agroalimentarios localizados". 2006.2 Disponible en: <http:www.cybercable.tm.fr/ jarmah/public html/DENIS4.htm\#2>. Acceso en: 16 jun. 2006 .

CAÑADA, J. S. Articulación espacial de la cadena agroalimentaria: Impacto de los procesos de globalización y reestructuración postfordista. In: GARCíA, P. B. La agricultura familiar en España. Estrategias adaptativas y políticas agropecuarias. Lleida: Universitat de Lleida, 1997. p. 149-179.

CAÑADA, J. S.; VÁZQUEZ, A. M. Quality certification, institutions and innovation in local agro-food systems: Protected designations of origin olive oil in Spain. Journal of rural studies, v. 21, p. 476-486. 2005.

SCHMITZ, H. Small shoemakers and fordist giants: Tale of a supercluster. World Development, v. 23, n. 1, p. 9. 1995.

SEN, A. K. Poverty and famines: an essay on entitlement and deprivation. New York: Clarendon Press; Oxford University Press, 1981. 
SEN, A. K. Hunger and entitlements: research for action. World Institute for Development Economics Research. Helsinki: United Nations University, 1987.

STIGLITZ, J.; SEN, A. K.; FITOUSSI, J. Vers de nouveaux systèmes de mesure. Paris: Odile Jacob, 2009.

SALCIDO, G. T. Los sistemas agroalimentarios locales. Capital social e instituciones. Una aproximación a la literatura reciente. In: ROMERO, M. E.; VIESCA, K. V. Teorías y problemas contemporáneos. Reflexiones desde la Ciencia Política. Mexico: Colima-UNAM, 2009. p. 153-173.

SALCIDO, G. T.; CHÁVEZ, H. A. R.; LEGLISE, M. del R. P. Los sistemas agroalimentarios localizados. Nuevas perspectivas para su análisis. In: SALCIDO, G. T.; CHÁVEZ, H. A. R.; LEGLISE, M. del R. P. Los sistemas agroalimentarios localizados en México. Desafíos para el desarrollo rural y la seguridad alimentaria. México: UNAM-CEIICH, p. 9-35. 2011.

WALLERSTEIN, I. M. El moderno sistema mundial. 5a ed. México: Siglo Veintiuno, 1989.

WINTER, M. Embeddedness, the new food economy and defensive localism. Journal of Rural Studies, v. 19, n. 1, p. 23-32. 2003. 